\title{
Reliability Based Bridging of the Gap between System's Safety Factors Associated with Different Failure Modes
}

\author{
W. Verhaeghe $\mathrm{e}^{\mathrm{a}, *}$, I. Elishakoff $\mathrm{f}^{\mathrm{b}}$ \\ ${ }^{a}$ Dept. of Mechanical Engineering, KU Leuven, B3001 Heverlee, Belgium \\ ${ }^{b}$ Dept. of Ocean and Mechanical Engineering, Florida Atlantic University, Boca Raton, \\ FL33431, USA
}

\begin{abstract}
The paper presents a study of the relation between safety factors corresponding to different failure modes in a mechanical system. The failure modes considered are displacement exceedance, yielding and buckling. A link between the safety factors is found using the reliability analysis. In particular, by postulating equal reliability against the considered failure modes, we derive the relation between the safety factors in three typical engineering examples.
\end{abstract}

Keywords: reliability, system failure, buckling

\section{Introduction}

Current practice of engineering is heavily based upon safety factors. On the other hand there is a strong sentiment in the engineering profession that one has to employ design that directly utilizes advances in modern uncertainty analyses, like probabilistic methodology that is based on the concept of reliability, or the fuzzy-sets based analysis that utilizes membership functions, or anti-optimization treatment that looks at worst case scenarios. Probabilistic design is apparently the most developed engineering tool currently. The current state of the art in structural reliability is discussed by several authors. The readers may consult with books by $[10,11,5]$.

This paper deals with the central issue of the reliability based safety factors, rather than the arbitrarily assigned ones, when two failure modes are available. As an example let us review a guideline found in a Russian textbook on strength of materials problems. Miroliubov et al [9] stress that the safety factor for stability "is always somewhat greater than the basic safety factor for strength, since during the analysis of compressed columns on stability one has to take into account additional unavoidable circumstances (eccentricity of load application, initial curvature and inhomogeneity of column's material) that facilitate the

\footnotetext{
* Corresponding author

Email addresses: wim.verhaeghe@mech.kuleuven.be (W. Verhaeghe), elishako@fau.edu (I. Elishakoff)
} 
bending. Large eccentricities and initial curvatures are analyzed specially, but the small ones, that are dependent on slenderness, are taken into account via increase of the safety factor against stability loss. Usually the following values are taken: for steel $n_{s t}=1.8-3$; for cast iron $n_{s t}=5-5.5$; for wood $n_{s t}=$ $2.8-3.2 . "$

Furthermore, there appears to be no consensus on required safety factors for stability. Izkovich [6] notes that the value of the "required stability safety factor depends basically on the utilization of the designed column and its material. Thus, for steel columns they take $n_{s t}=1.7-2$ in civil engineering; $n_{s t}=3.5-5$ in mechanical engineering. For cast iron columns in average $n_{s t}=5$; for wood in average $n_{s t}=3 . "$

The interplay between reliability and safety factors was discussed by numerous authors in past decades. For the extensive summary one can consult apparently the single book on this topic [3]. This book calls for "peaceful coexistence" between reliability concept and safety factors. Specifically, this could be interpreted as the reliability based assignment of safety factors without discarding the latter. Some recent codes practically implement such a tolerant approach: EUROCODE introduces both safety factors and reliability implying that one can make a choice between these competing philosophies. Indeed, in section 3.5 of EN1990 the design engineer finds the following statements:

(a) "The requirements ... should be achieved by partial factor (W.V.: safety factors) method ..."

(b) "As an alternative, a design directly based on probabilistic methods may be used."

The EUROCODE as well as the Load and Resistance Factor Design (LRFD) of the American Institute of Steel Construction introduce safety factors (partial factors) based on a reliability assessment. In particular, the EUROCODE details the background of the partial factor method in Annex 6 of EN1990. An overview of the developments and an outlook on the next steps to undertake in the LRFD code is given by Chen [2] and by Ellingwood [4]. Practical application of the LRFD code is explained in [1].

In this paper we deal with some academical engineering problems that exhibit two or more failure modes from following set: stress levels exceeding the yield stress of the material, displacement of part of the structure exceeding a prescribed maximum and buckling of the structure. Engineers usually assign different safety factors to these modes. The keen reader however may not grasp why different safety factors are assigned against these failure modes. This paper attempts to shed some light on this topic.

\section{Different failure modes, different safety factors?}

Let us consider class of beam and truss problems in which three failure modes are studied. In particular we look at following conditions: the maximal stress should not exceed the yield stress $\sigma_{y}$, some displacement should not exceed a maximum $\Delta l_{\max }$, and additionally a beam must not buckle (buckling is 
often analysed using so called buckling curves, see i.e. [8]). The corresponding mathematical conditions are:

$$
\begin{array}{r}
\sigma=\frac{P}{A} \leq \sigma_{\text {all }} \\
\Delta l \leq \Delta l_{\text {all }} \\
P \leq N_{\text {all }}
\end{array}
$$

with $P$ and $A$ being the load and cross-sectional area of the beam, respectively. Quantities $\sigma_{\text {all }}, \Delta l_{\text {all }}$ and $N_{\text {all }}$ are the allowable stress, displacement and axial force, respectively:

$$
\begin{aligned}
\sigma_{\text {all }} & =\frac{\sigma_{y}}{n_{1}} \\
\Delta l_{\text {all }} & =\frac{\Delta l_{\text {max }}}{n_{2}} \\
N_{\text {all }} & =\frac{N_{\text {critical }}}{n_{3}}=\frac{1}{n_{3}} \frac{\pi^{2} E I}{l^{2}}
\end{aligned}
$$

with $n_{1}, n_{2}$ and $n_{3}$, the associated safety factors for the stress, displacement and buckling failure mode, respectively. The study investigates the relationship between these safety factors. It is shown that the concept of reliability allows one to assign safety factors in a unified manner. This not necessarily implies equal safety factors, but as will be shown later does not exclude that option.

\section{Beam under compression load}

The following study is based on an example problem due to Volmir [14]. A simply supported steel beam of length $l=1 \mathrm{~m}$ with rectangular cross section with a height equal to three times the width $(h=3 b)$, is axially loaded by a compression force $P$. Only the yield and buckling failure mode are applied to this problem:

$$
\begin{array}{r}
\sigma=\frac{P}{A}=\frac{P}{3 b^{2}} \leq \sigma_{\text {all }} \\
P \leq N_{\text {all }}
\end{array}
$$

with $\sigma_{\text {all }}$ and $N_{\text {all }}$, the allowable stress and axial force, respectively, defined in Eq. (4) and (6).

\subsection{Random dimension $b$}

If the dimension $b$ is considered random, then the reliability of the structure, if only the yield and buckling constraints are considered, can be calculated as follows:

$$
R=\operatorname{Prob}\left\{\left(\sigma \leq \sigma_{y}\right) \cap\left(P \leq N_{\text {critical }}\right)\right\}
$$


where $\cap$ designates the operation of intersection. In terms of the random variable $b$ the reliability becomes:

$$
R=\operatorname{Prob}\left\{\left(\sqrt{\frac{P}{3 \sigma_{y}}} \leq b\right) \cap\left(\sqrt[4]{\frac{P 4 l^{2}}{\pi^{2} E}} \leq b\right)\right\}
$$

The following three cases can be encountered. In the first case the following inequality is satisfied:

$$
\sqrt{\frac{P}{3 \sigma_{y}}}>\sqrt[4]{\frac{P 4 l^{2}}{\pi^{2} E}}
$$

then the reliability is dominated by the yield constraint. In the second case we have:

$$
\sqrt[4]{\frac{P 4 l^{2}}{\pi^{2} E}}>\sqrt{\frac{P}{3 \sigma_{y}}}
$$

then the reliability is dominated by the buckling constraint. Finally, in the third case the following equality takes place:

$$
\sqrt{\frac{P}{3 \sigma_{y}}}=\sqrt[4]{\frac{P 4 l^{2}}{\pi^{2} E}}
$$

then either of the constraints can be used to find the reliability.

For the sake of simplicity let us assume a uniform distribution for $b$ between values $g$ and $h$. (The interested reader can find an analysis with different kind of distribution function in Appendix) This results in a probability density function:

$$
f_{b}= \begin{cases}0 & \text { if } b<g \text { or } b>h \\ \frac{1}{h-g} & \text { if } g \leq b \leq h\end{cases}
$$

so that the mean equals $E[b]=\int_{g}^{h} b /(h-g) \mathrm{d} b=(g+h) / 2$, and the standard deviation is

$$
d_{b}=\sqrt{E\left[(b-E[b])^{2}\right]}=\sqrt{\int_{g}^{h}\left(b-\frac{g+h}{2}\right)^{2} \frac{1}{h-g} \mathrm{~d} b}=\frac{h-g}{2 \sqrt{3}}
$$

The lower and upper bound on $b$ can be described by the mean and the standard deviation: $g=E[b]-\sqrt{3} d_{b}$ and $\mathrm{h}=E[b]+\sqrt{3} d_{b}$. The reliability for the first case is thus calculated as

$$
R_{1}= \begin{cases}0 & \text { if } \sqrt{\frac{P}{3 \sigma_{y}}}>h=E[b]+\sqrt{3} d_{b} \\ 1-\frac{\sqrt{\frac{P}{3 \sigma_{y}}}-E[b]+\sqrt{3} d_{b}}{2 \sqrt{3} d_{b}} & \text { if } g \leq \sqrt{\frac{P}{3 \sigma_{y}}} \leq h \\ 1 & \text { if } \sqrt{\frac{P}{3 \sigma_{y}}}<g=E[b]-\sqrt{3} d_{b}\end{cases}
$$


The reliability for the second case is evaluated as

$$
R_{2}= \begin{cases}0 & \text { if } \sqrt[4]{\frac{P 4 l^{2}}{\pi^{2} E}}>h=E[b]+\sqrt{3} d_{b} \\ 1-\frac{\sqrt[4]{\frac{P 4 l^{2}}{\pi^{2} E}}-E[b]+\sqrt{3} d_{b}}{2 \sqrt{3} d_{b}} & \text { if } g \leq \sqrt[4]{\frac{P 4 l^{2}}{\pi^{2} E}} \leq h \\ 1 & \text { if } \sqrt[4]{\frac{P 4 l^{2}}{\pi^{2} E}}<g=E[b]-\sqrt{3} d_{b}\end{cases}
$$

The mean value of the stress is

$$
E[\Sigma]=\frac{P}{3} E\left[\frac{1}{b^{2}}\right]=\frac{P}{3} \frac{1}{(E[b])^{2}-3 d_{b}^{2}}
$$

since

$$
E\left[\frac{1}{b^{2}}\right]=\int_{E[b]-\sqrt{3} d_{b}}^{E[b]+\sqrt{3} d_{b}} \frac{1}{b^{2}} \frac{1}{2 \sqrt{3} d_{b}} d b=\frac{1}{(E[b])^{2}-3 d_{b}^{2}}
$$

Multiplying the numerator and denominator of the middle part of Eq. (16) by $\sqrt{E[\Sigma] \text { yields }}$

$$
R_{1}=1-\frac{\sqrt{\frac{P}{3 \sigma_{y}}} \sqrt{E[\Sigma]}-E[b] \sqrt{E[\Sigma]}+\sqrt{3} d_{b} \sqrt{E[\Sigma]}}{2 \sqrt{3} d_{b} \sqrt{E[\Sigma]}}
$$

Defining the central safety factor for the stress condition

$$
n_{1}=\sigma_{y} / E[\Sigma]
$$

and noting that the coefficient of variation of $b$ is $\nu_{b}=d_{b} / E[b]$, Eq. (20) becomes:

$$
R_{1}=1-\frac{\sqrt{\frac{P}{3 n_{1}}}-\sqrt{\frac{P}{3\left(1-3 \nu_{b}^{2}\right)}}+\sqrt{\frac{P 3 \nu_{b}^{2}}{3\left(1-3 \nu_{b}^{2}\right)}}}{\sqrt{\frac{P 12 \nu_{b}^{2}}{3\left(1-3 \nu_{b}^{2}\right)}}}=1-\frac{\sqrt{\frac{1}{n_{1}}}-\sqrt{\frac{1}{1-3 \nu_{b}^{2}}}+\sqrt{\frac{3 \nu_{b}^{2}}{1-3 \nu_{b}^{2}}}}{\sqrt{\frac{12 \nu_{b}^{2}}{1-3 \nu_{b}^{2}}}}
$$

The expression for the target safety factor $n_{1}$ as a function of the target reliability $R_{1}=r_{1}$ is

$$
n_{1}=\left[\left(1-r_{1}\right) \sqrt{\frac{12 \nu_{b}^{2}}{1-3 \nu_{b}^{2}}}+\sqrt{\frac{1}{1-3 \nu_{b}^{2}}}-\sqrt{\frac{3 \nu_{b}^{2}}{1-3 \nu_{b}^{2}}}\right]^{-2}
$$

On the other hand, the mean value of the critical load equals

$$
\begin{aligned}
E\left[N_{\text {critical }}\right] & =E\left[\frac{\pi^{2} E b^{4}}{4 l^{2}}\right]=\frac{\pi^{2} E}{4 l^{2}} E\left[b^{4}\right] \\
& =\frac{\pi^{2} E}{4 l^{2}} \frac{1}{10 \sqrt{3} d_{b}}\left[10(E[b])^{4} \sqrt{3} d_{b}+60(E[b])^{2} \sqrt{3} d_{b}^{3}+18 \sqrt{3} d_{b}^{5}\right]
\end{aligned}
$$


Dividing the numerator and denominator of the middle part of Eq. (17) by $\sqrt[4]{E\left[N_{\text {critical }}\right]}$ results in

$$
R_{2}=1-\frac{\sqrt[4]{\frac{4 l^{2}}{\pi^{2} E} \frac{P}{E\left[N_{\text {critical }}\right]}}-\frac{E[b]}{\sqrt[4]{E\left[N_{\text {critical }}\right]}}+\frac{\sqrt{3} d_{b}}{\sqrt[4]{E\left[N_{\text {critical }}\right]}}}{\frac{2 \sqrt{3} d_{b}}{\sqrt[4]{E\left[N_{\text {critical }}\right]}}}
$$

Introducing the central safety factor against buckling

$$
n_{3}=E\left[N_{\text {critical }}\right] / P
$$

leads to:

$$
R_{2}=1-\frac{\sqrt[4]{\frac{1}{n_{3}}}-\sqrt[4]{\frac{1}{Q}}+\sqrt[4]{\frac{9 \nu_{b}^{4}}{Q}}}{\sqrt[4]{\frac{144 \nu_{b}^{4}}{Q}}}, \quad Q=\frac{E\left[N_{\text {critical }}\right]}{(E[b])^{4}} \frac{4 l^{2}}{\pi^{2} E}=\frac{1}{5}\left(5+30 \nu_{b}^{2}+9 \nu_{b}^{4}\right)
$$

The expression for the target safety factor $n_{3}$ as a function of the target reliability $R_{2}=r_{2}$ is

$$
n_{3}=\left[\left(1-r_{2}\right) \sqrt[4]{\frac{144 \nu_{b}^{4}}{Q}}+\sqrt[4]{\frac{1}{Q}}-\sqrt[4]{\frac{9 \nu_{b}^{4}}{Q}}\right]^{-4}
$$

We postulate that the structure should possess the uniform reliability against each mode of failure, namely if $r_{1}=r_{2}$, then the following relationship between the safety factors $n_{1}$ and $n_{3}$ is found:

$$
n_{1}=\left[\frac{1}{\sqrt{1-3 \nu_{b}^{2}}}\left(\sqrt[4]{\frac{Q}{n_{3}}}-1+\sqrt[4]{9 \nu_{b}^{4}}\right)+\sqrt{\frac{1}{1-3 \nu_{b}^{2}}}-\sqrt{\frac{3 \nu_{b}^{2}}{1-3 \nu_{b}^{2}}}\right]^{-2}
$$

or formulated differently:

$$
n_{3}=\left[\frac{1}{\sqrt[4]{Q}}\left(\sqrt{\frac{1-3 \nu_{b}^{2}}{n_{1}}}-1+\sqrt{3 \nu_{b}^{2}}\right)+\sqrt[4]{\frac{1}{Q}}-\sqrt[4]{\frac{9 \nu_{b}^{4}}{Q}}\right]^{-4}
$$

Let us consider a numerical example. Following numerical values are adopted: $F=500 k N, \sigma_{y}=1100 M P a, E=210 G P a$. We assume the lower bound $g=0.01 \mathrm{~m}$ and the upper bound $h=0.035 \mathrm{~m}$ for the uniform distribution of $b$. Then the following values are calculated:

$$
\begin{aligned}
E[b] & =0.0225 m \\
d_{b} & =0.00722 m \\
\nu_{b} & =0.321
\end{aligned}
$$




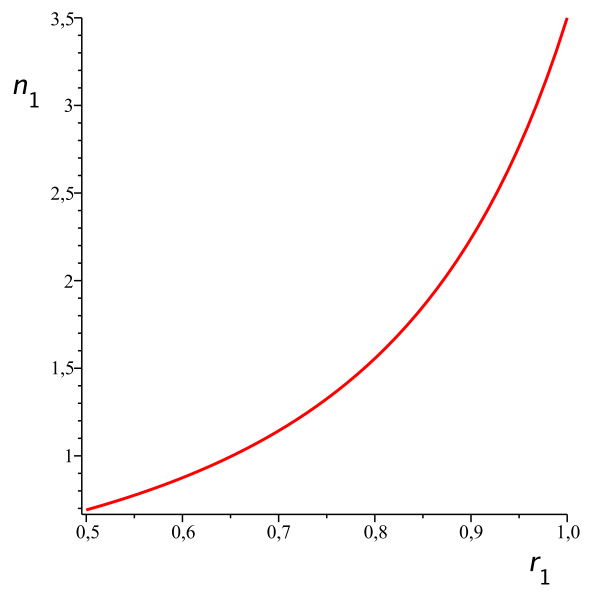

Figure 1: The safety factor $n_{1}$ as a function of the required reliability $r_{1}$

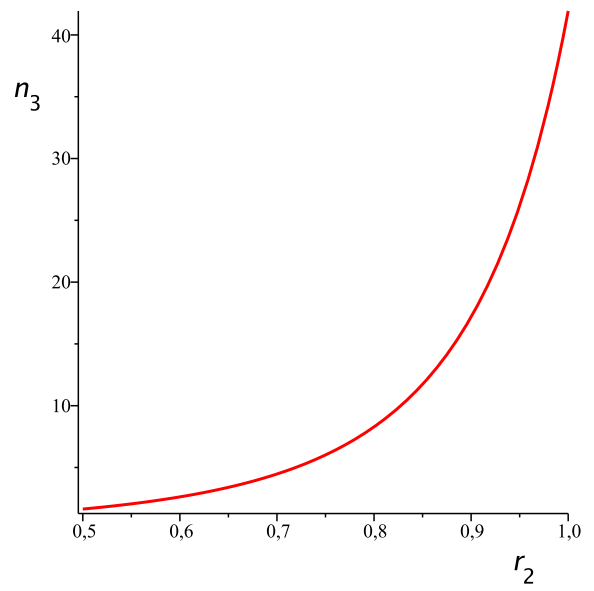

Figure 2: The safety factor $n_{3}$ as a function of the required reliability $r_{2}$ 


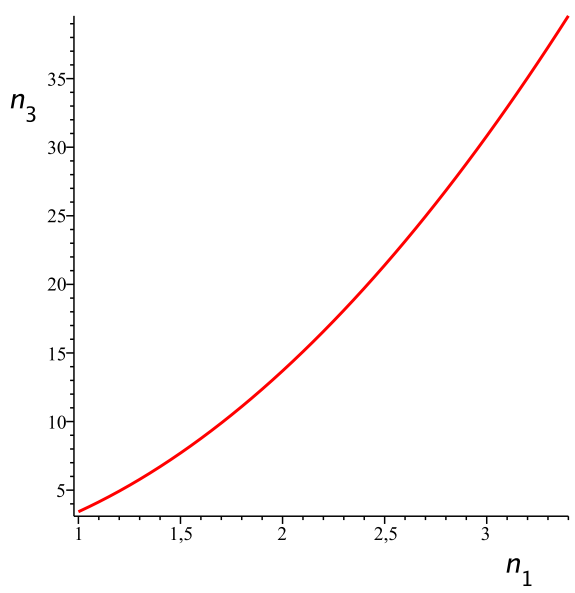

Figure 3: The safety factor $n_{3}$ as a function of the safety factor $n_{1}$ for the numerical example

Figure 1 shows the safety factor $n_{1}$ as a function of target reliability $r_{1}$ corresponding to Eq. (23). Figure 2 shows the safety factor $n_{3}$ as a function of target reliability $r_{2}$ corresponding to Eq. (28). Figure 3 shows the safety factor $n_{3}$ as a function of the safety factor $n_{1}$ on the condition that the same reliability is required as expressed by Eq. (30). From the figures and the equations following example values are found:

- If one would demand the same reliability 0.9 for both failure modes, then the required safety factors would be $n_{1}=2.24$ and $n_{3}=17.2$.

- If one would demand the same reliability 0.99 for both failure modes, then the required safety factors would be $n_{1}=3.33$ and $n_{3}=38$.

As is seen in both cases, the different safety factors are obtained, for different failure modes. Without having an "intermediary" in the form of reliability, the design may have turned out to constitute either overdesign or underdesign.

\section{Truss Problem With Three Potential Failure Modes}

A structure as depicted in Figure 4 is analysed in Example Problem 7.11 of the book by Vinokurov [13]. The horizontal member is loaded in tension $N_{A B}=1.73 \mathrm{~F}$ and the diagonal member is loaded in compression $N_{B C}=2 \mathrm{~F}$. The total structure will fail if either the tensioned member will yield, the vertical displacement $u_{B y}$ exceeds a maximum or the compressed member will buckle or both. To avoid these failures, following conditions are set forth: 


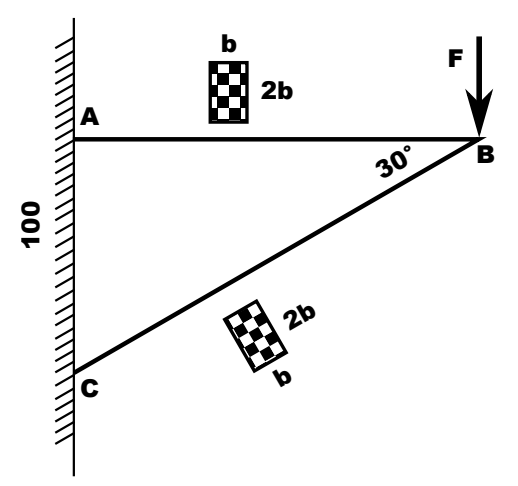

Figure 4: Problem description

$$
\begin{array}{r}
\sigma_{A B}=\frac{N_{A B}}{A_{A B}}=\frac{1.73 F}{2 b^{2}}=0.865 \frac{F}{b^{2}} \leq \sigma_{\text {all }} \\
u_{B y}=\frac{F}{E A}\left(\frac{-3}{16 l_{B C}^{2}} \frac{1}{\frac{1}{l_{A B}}+\frac{3}{4 l_{B C}}}+\frac{1}{4 l_{B C}}\right)^{-1}=\frac{F}{E 2 b^{2}}(0.7578)^{-1} \leq \Delta l_{\text {all }} \\
N_{B C} \leq N_{\text {all }}
\end{array}
$$

with $\sigma_{\text {all }}, \Delta l_{\text {all }}$ and $N_{\text {all }}$, the allowable stress, displacement and axial force, respectively, as defined in Eq. (4), (5) and (6).

\subsection{Random Dimension $b$}

If the dimension $b$ is considered random, then the reliability of the structure can be calculated as follows:

$$
R=\operatorname{Prob}\left\{\left(\sigma \leq \sigma_{y}\right) \cap\left(u_{B y} \leq \Delta l_{\max }\right) \cap\left(N_{B C} \leq N_{\text {critical }}\right)\right\}
$$

In terms of the random variable $b$ reliability becomes:

$$
R=\operatorname{Prob}\left\{\left(\sqrt{\frac{0.865 F}{\sigma_{y}}} \leq b\right) \cap\left(\sqrt{\frac{F}{1.5156 E \Delta l_{\max }}} \leq b\right) \cap\left(\sqrt[4]{\frac{2 F 6 l_{B C}^{2}}{\pi^{2} E}} \leq b\right)\right\}
$$

The following four cases can be encountered:

1. $\sqrt{\frac{0.865 F}{\sigma_{y}}}>\max \left(\sqrt{\frac{F}{1.5156 E \Delta l_{\max }}}, \sqrt[4]{\frac{2 F 6 l_{L_{C}}^{2}}{\pi^{2} E}}\right)$, then the reliability is determined by the tension constraint.

2. $\sqrt{\frac{F}{1.5156 E \Delta l_{\max }}}>\max \left(\sqrt{\frac{0.865 F}{\sigma_{y}}}, \sqrt[4]{\frac{2 F 6 l_{B C}^{2}}{\pi^{2} E}}\right)$, then the reliability is decided by the displacement constraint. 
3. $\sqrt[4]{\frac{2 F 6 l_{B C}^{2}}{\pi^{2} E}}>\max \left(\sqrt{\frac{0.865 F}{\sigma_{y}}}, \sqrt{\frac{F}{1.5156 E \Delta l_{\max }}}\right)$, then the reliability is evaluated by the buckling constraint.

4. $\sqrt{\frac{0.865 F}{\sigma_{y}}}=\sqrt{\frac{F}{1.5156 E \Delta l_{\max }}}=\sqrt[4]{\frac{2 F 6 l_{B C}^{2}}{\pi^{2} E}}$, then either constraint can be used to find the reliability.

Let us again assume a uniform distribution for $b$ between $g$ and $h$. Thus, the mean $E[b]=(g+h) / 2$ and the standard deviation $d_{b}=(h-g) / 2 \sqrt{3}$. The reliability against the failure in tension is calculated as

$$
R_{1}= \begin{cases}0 & \text { if } \sqrt{\frac{0.865 F}{\sigma_{y}}}>h=E[b]+\sqrt{3} d_{b} \\ 1-\frac{\sqrt{\frac{0.885 F}{\sigma_{y}}}-E[b]+\sqrt{3} d_{b}}{2 \sqrt{3} d_{b}} & \text { if } g \leq \sqrt{\frac{0.865 F}{\sigma_{y}}} \leq h \\ 1 & \text { if } \sqrt{\frac{0.865 F}{\sigma_{y}}}<g=E[b]-\sqrt{3} d_{b}\end{cases}
$$

The against the excessive displacement is calculated as

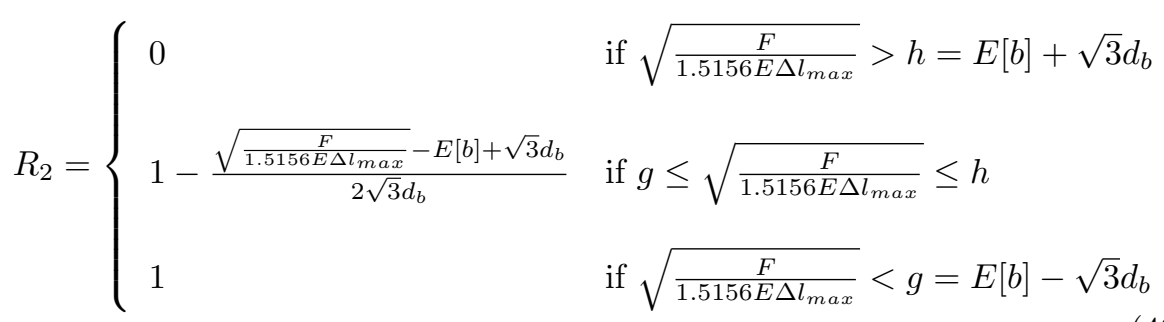

The reliability against buckling reads

$$
R_{3}= \begin{cases}0 & \text { if } \sqrt[4]{\frac{2 F 6 l_{B C}^{2}}{\pi^{2} E}}>h=E[b]+\sqrt{3} d_{b} \\ 1-\frac{\sqrt[4]{\frac{2 F 6 l_{B C}^{2}}{\pi^{2} E}}-E[b]+\sqrt{3} d_{b}}{2 \sqrt{3} d_{b}} & \text { if } g \leq \sqrt[4]{\frac{2 F 6 l_{B C}^{2}}{\pi^{2} E}} \leq h \\ 1 & \text { if } \sqrt[4]{\frac{2 F 6 l_{B C}^{2}}{\pi^{2} E}}<g=E[b]-\sqrt{3} d_{b}\end{cases}
$$

The mean of the stress is found to be

$$
E[\Sigma]=0.865 F E\left[\frac{1}{b^{2}}\right]=0.865 F \frac{1}{(E[b])^{2}-3 d_{b}^{2}}
$$

Multiplying the numerator and denominator of the middle part of Eq. (39) by $\sqrt{E[\Sigma]}$ yields

$$
R_{1}=1-\frac{\sqrt{\frac{0.865 F}{\sigma_{y}}} \sqrt{E[\Sigma]}-E[b] \sqrt{E[\Sigma]}+\sqrt{3} d_{b} \sqrt{E[\Sigma]}}{2 \sqrt{3} d_{b} \sqrt{E[\Sigma]}}
$$


Defining the central safety factor as in Eq. (21) results in:

$$
R_{1}=1-\frac{\sqrt{\frac{0.865 F}{n_{1}}}-\sqrt{\frac{0.865 F}{1-3 \nu_{b}^{2}}}+\sqrt{\frac{0.865 F 3 \nu_{b}^{2}}{1-3 \nu_{b}^{2}}}}{\sqrt{\frac{0.865 F 12 \nu_{b}^{2}}{1-3 \nu_{b}^{2}}}}=1-\frac{\sqrt{\frac{1}{n_{1}}}-\sqrt{\frac{1}{1-3 \nu_{b}^{2}}}+\sqrt{\frac{3 \nu_{b}^{2}}{1-3 \nu_{b}^{2}}}}{\sqrt{\frac{12 \nu_{b}^{2}}{1-3 \nu_{b}^{2}}}}
$$

The expression for the safety factor $n_{1}$ as a function of the target reliability $r_{1}$ is

$$
n_{1}=\left[\left(1-R_{1}\right) \sqrt{\frac{12 \nu_{b}^{2}}{1-3 \nu_{b}^{2}}}+\sqrt{\frac{1}{1-3 \nu_{b}^{2}}}-\sqrt{\frac{3 \nu_{b}^{2}}{1-3 \nu_{b}^{2}}}\right]^{-2}
$$

Next, noting that the mean of the vertical displacement is

$$
E\left[u_{B y}\right]=\frac{F}{1.5156 E} E\left[\frac{1}{b^{2}}\right]=\frac{F}{1.5156 E} \frac{1}{(E[b])^{2}-3 d_{b}^{2}}
$$

Multiplying the numerator and denominator of the middle part of Eq. (40) by $\sqrt{E\left[u_{B y}\right]}$ results in

$$
R_{2}=1-\frac{\sqrt{\frac{F}{1.5156 E \Delta l_{\max }}} \sqrt{E\left[u_{B y}\right]}-E[b] \sqrt{E\left[u_{B y}\right]}+\sqrt{3} d_{b} \sqrt{E\left[u_{B y}\right]}}{2 \sqrt{3} d_{b} \sqrt{E\left[u_{B y}\right]}}
$$

Defining the central safety factor

$$
n_{2}=\Delta l_{\max } / E\left[u_{B y}\right]
$$

and utilizing the coefficient of variation $\nu_{b}$ results in

$$
\begin{aligned}
R_{2} & =1-\frac{\sqrt{\frac{F}{1.5156 E} \frac{1}{n_{2}}}-\sqrt{\frac{F}{1.5156 E\left(1-3 \nu_{b}^{2}\right)}}+\sqrt{\frac{F 3 \nu_{b}^{2}}{1.5156 E\left(1-3 \nu_{b}^{2}\right)}}}{\sqrt{\frac{F 12 \nu_{b}^{2}}{1.5156 E\left(1-3 \nu_{b}^{2}\right)}}} \\
& =1-\frac{\sqrt{\frac{1}{n_{2}}}-\sqrt{\frac{1}{1-3 \nu_{b}^{2}}}+\sqrt{\frac{3 \nu_{b}^{2}}{1-3 \nu_{b}^{2}}}}{\sqrt{\frac{12 \nu_{b}^{2}}{1-3 \nu_{b}^{2}}}}
\end{aligned}
$$

The expression for the safety factor $n_{2}$ as a function of the target reliability $R_{2}$ is

$$
n_{2}=\left[\left(1-r_{2}\right) \sqrt{\frac{12 \nu_{b}^{2}}{1-3 \nu_{b}^{2}}}+\sqrt{\frac{1}{1-3 \nu_{b}^{2}}}-\sqrt{\frac{3 \nu_{b}^{2}}{1-3 \nu_{b}^{2}}}\right]^{-2}
$$

The last two equations coincide with Eq. (44) and (45), respectively. This is a result of both constraints depending in the same quadratic order on the random dimension $b$. The different scaling coefficients $0.865 F$ and $\frac{F}{1.5156 E}$ cancel out in 
the derivation of the respective relations between the reliability and the safety factor.

It is instructive to search the relation between $R_{3}$ and $n_{3}$; we take into account that the mean of the critical load is

$$
\begin{aligned}
E\left[N_{\text {critical }}\right] & =E\left[\frac{\pi^{2} E b^{4}}{6 l_{B C}^{2}}\right]=\frac{\pi^{2} E}{6 l_{B C}^{2}} E\left[b^{4}\right] \\
& =\frac{\pi^{2} E}{6 l_{B C}^{2}} \frac{1}{10 \sqrt{3} d_{b}}\left[10(E[b])^{4} \sqrt{3} d_{b}+60(E[b])^{2} \sqrt{3} d_{b}^{3}+18 \sqrt{3} d_{b}^{5}\right]
\end{aligned}
$$

and rewrite Eq. (41) in the form

$$
R_{3}=1-\frac{\sqrt[4]{\frac{6 l_{B C}^{2}}{\pi^{2} E} \frac{2 F}{E\left[N_{\text {critical }}\right]}}-\frac{E[b]}{\sqrt[4]{E\left[N_{\text {critical }}\right]}}+\frac{\sqrt{3} d_{b}}{\sqrt[4]{E\left[N_{\text {critical }}\right]}}}{\frac{2 \sqrt{3} d_{b}}{\sqrt[4]{E\left[N_{\text {critical }}\right]}}}
$$

Taking into account the definition (26), we arrive at:

$$
R_{3}=1-\frac{\sqrt[4]{\frac{1}{n_{3}}}-\sqrt[4]{\frac{1}{Q}}+\sqrt[4]{\frac{9 \nu_{b}^{4}}{Q}}}{\sqrt[4]{\frac{144 \nu_{b}^{4}}{Q}}}, \quad Q=\frac{E\left[N_{\text {critical }}\right]}{(E[b])^{4}} \frac{6 l_{B C}^{2}}{\pi^{2} E}=\frac{1}{5}\left(5+30 \nu_{b}^{2}+9 \nu_{b}^{4}\right)
$$

The expression for the safety factor $n_{3}$ as a function of the target reliability $r_{3}$ is

$$
n_{3}=\left[\left(1-r_{3}\right) \sqrt[4]{\frac{144 \nu_{b}^{4}}{Q}}+\sqrt[4]{\frac{1}{Q}}-\sqrt[4]{\frac{9 \nu_{b}^{4}}{Q}}\right]^{-4}
$$

If the same reliability is demanded for all constraints then the following relationship between $n_{1}, n_{2}$ and $n_{3}$ is found:

$$
n_{1}=n_{2}=\left[\frac{1}{\sqrt{1-3 \nu_{b}^{2}}}\left(\sqrt[4]{\frac{Q}{n_{3}}}-1+\sqrt[4]{9 \nu_{b}^{4}}\right)+\sqrt{\frac{1}{1-3 \nu_{b}^{2}}}-\sqrt{\frac{3 \nu_{b}^{2}}{1-3 \nu_{b}^{2}}}\right]^{-2}
$$

or, if formulated differently, we get:

$$
\begin{aligned}
n_{3} & =\left[\frac{1}{\sqrt[4]{Q}}\left(\sqrt{\frac{1-3 \nu_{b}^{2}}{n_{1}}}-1+\sqrt{3 \nu_{b}^{2}}\right)+\sqrt[4]{\frac{1}{Q}}-\sqrt[4]{\frac{9 \nu_{b}^{4}}{Q}}\right]^{-4} \\
& =\left[\frac{1}{\sqrt[4]{Q}}\left(\sqrt{\frac{1-3 \nu_{b}^{2}}{n_{2}}}-1+\sqrt{3 \nu_{b}^{2}}\right)+\sqrt[4]{\frac{1}{Q}}-\sqrt[4]{\frac{9 \nu_{b}^{4}}{Q}}\right]^{-4}
\end{aligned}
$$

Let us consider a numerical example. Following numerical values are adopted: $F=20 k N, \sigma_{y}=600 M P a, E=210 G P a$. Assume the lower bound $g=0.005 m$ 
and the upper bound $h=0.009 \mathrm{~m}$ for the uniform distribution of $b$. Then the following values are calculated:

$$
\begin{aligned}
E[b] & =0.007 \mathrm{~m} \\
d_{b} & =0.001155 \mathrm{~m} \\
\nu_{b} & =0.165
\end{aligned}
$$

If one would demand the same reliability 0.9 for all three failure modes, then the required safety factors would be $n_{1}=n_{2}=1.54$ and $n_{3}=3.29$. For a target reliability equal to 0.99 the required safety factors would be $n_{1}=n_{2}=1.77$ and $n_{3}=4.33$. In the original example in Ref. [13] the safety factors suggested by Vinokurov are $n_{1}=2.5$ and $n_{3}=4$. These safety factors do not satisfy Eq. (55). For the considered random $b$, this safety factor $n_{1}$ would result in a very conservative design with respect to the tension constraint since the maximum safety factor corresponding to $R_{1}=1$ is $n_{1}=1.8$. On the other hand, the maximum safety factor $n_{3}$ corresponding to $R_{3}=1$ is $n_{3}=4.47$. The suggested safety factor $n_{3}=4$ corresponds to an $R_{3}=0.964$. If the same reliability $R_{1}$ is requested, then $n_{1}=1.7$ should be taken, which is not far from the Vinokurov's [13] values.

\subsection{Safety Factors in the Case of a Random Load F}

If the load $F$ is considered random, then the reliability of the structure Eq. (37) can be expressed in terms of the random $F$ :

$$
R=\operatorname{Prob}\left\{\left(F \leq \frac{\sigma_{y} b^{2}}{0.865}\right) \cap\left(F \leq 1.5156 b^{2} E \Delta l_{\max }\right) \cap\left(F \leq \frac{1}{2} N_{\text {critical }}\right)\right\}
$$

The following four cases can be encountered:

(a) $\frac{\sigma_{y} b^{2}}{0.865}<\min \left(1.5156 b^{2} E \Delta l_{\max }, \frac{1}{2} N_{\text {critical }}\right)$, then the reliability is dominated by the tension constraint.

(b) $1.5156 b^{2} E \Delta l_{\max }<\min \left(\frac{\sigma_{y} b^{2}}{0.865}, \frac{1}{2} N_{\text {critical }}\right)$, then the reliability is dominated by the displacement constraint.

(c) $\frac{1}{2} N_{\text {critical }}<\min \left(\frac{\sigma_{y} b^{2}}{0.865}, 1.5156 b^{2} E \Delta l_{\max }\right)$, then the reliability is dominated by the buckling constraint.

(d) $\frac{\sigma_{y} b^{2}}{0.865}=1.5156 b^{2} E \Delta l_{\text {max }}=\frac{1}{2} N_{\text {critical }}$, then either constraint can be used to find the reliability.

Let us assume a uniform distribution for $F$ in the similar fashion as was done for $b$ in the above section. The reliability for all the cases can be written in a unified form

$$
R= \begin{cases}0 & \text { if } x<g=E[F]-\sqrt{3} d_{F} \\ \frac{x-E[F]+\sqrt{3} d_{F}}{2 \sqrt{3} d_{F}} & \text { if } g \leq x \leq h \\ 1 & \text { if } x>h=E[F]+\sqrt{3} d_{F}\end{cases}
$$


where $x$ needs to be replaced by $\frac{\sigma_{y} b^{2}}{0.865}, 1.5156 b^{2} E \Delta l_{\text {max }}$ or $\frac{1}{2} N_{\text {critical }}$ to find the reliability for the first, second and third cases, respectively.

Taking into account that the mean value of the stress

$$
E[\Sigma]=\frac{0.865}{b^{2}} E[F]
$$

and dividing the numerator and denominator of the middle part of Eq. (61) for the first case by $E[\Sigma]$ gives

$$
R_{1}=\frac{\frac{\sigma_{y}}{E[\Sigma]} \frac{b^{2}}{0.865}-\frac{b^{2}}{0.865}+\sqrt{3} \frac{b^{2}}{0.865} \nu_{F}}{2 \sqrt{3} \frac{b^{2}}{0.865} \nu_{F}}
$$

Taking into account the definition of the central safety factor $n_{1}$ in Eq. (21) and noting that the coefficient of variation of the force is $\nu_{F}=\frac{d_{F}}{E[F]}$, gives the following result:

$$
R_{1}=\frac{n_{1}-1+\sqrt{3} \nu_{F}}{2 \sqrt{3} \nu_{F}}
$$

The expression for the safety factor $n_{1}$ as a function of the target reliability $r_{1}$ is

$$
n_{1}=2 \sqrt{3} \nu_{F} r_{1}+1-\sqrt{3} \nu_{F}
$$

The same calculations but considering the mean of the vertical displacement of node B $E\left[u_{B y}\right]=\left(1 / 1.5156 b^{2} E\right) E[F]$ can be performed for the second case (dominant displacement constraint) with the central safety factor $n_{2}$ defined as in Eq. (48). The result of these calculations also yield Eq. (64) and (65), but with the variables $n_{1}, R_{1}, r_{1}$ replaced by $n_{2}, R_{2}, r_{2}$, respectively.

On the other hand, dividing the numerator and denominator of the middle part of Eq. (61) for the third case by $E\left[N_{B C}\right]=2 E[F]$ gives

$$
R_{3}=\frac{\frac{N_{\text {critical }}}{2 E\left[N_{B C}\right]}-\frac{E[F]}{2 E[F]}+\sqrt{3} \frac{d_{F}}{2 E[F]}}{2 \sqrt{3} \frac{d_{F}}{2 E[F]}}
$$

with the central safety factor $n_{3}$ this time defined as

$$
n_{3}=N_{\text {critical }} / E\left[N_{B C}\right]
$$

we also find Eq. (64) and (65), but with $n_{1}, R_{1}, r_{1}$ replaced by $n_{3}, R_{3}, r_{3}$.

If the same reliability is demanded for all constraints then the following relationship between $n_{1}, n_{2}$ and $n_{3}$ is found:

$$
n_{1}=n_{2}=n_{3}
$$

Let us consider a numerical example. Following numerical values are adopted: $b=0.01 \mathrm{~m}, \sigma_{y}=600 M P a, E=210 G P a$. Assume the lower bound $g=30 k N$ 


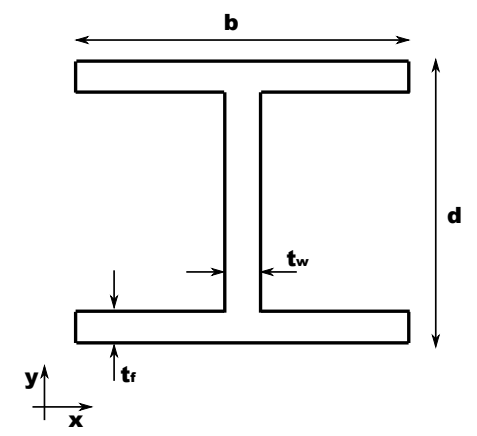

Figure 5: The dimensions of the I-beam's cross-section

and the upper bound $h=70 \mathrm{kN}$ for the uniform distribution of $F$. Then the following values are calculated:

$$
\begin{aligned}
E[F] & =50 k N \\
d_{F} & =11.547 k N \\
\nu_{F} & =0.23094
\end{aligned}
$$

If one would demand the same reliability 0.9 for all failure modes, then the required safety factors would be $n_{1}=n_{2}=n_{3}=1.32$. Instead, if one would demand the same reliability 0.99 for the three failure modes, then we find $n_{1}=$ $n_{2}=n_{3}=1.39$. In the original example in [13] the suggested safety factors are $n_{1}=2.5$ and $n_{3}=4$. For the considered random variable $F$, this would result in a very conservative design. Furthermore, these safety factors do not satisfy Eq. (68). Based on the previous probabilistic analysis, it seems that the reliability based on the suggested safety factor for the buckling resistance is higher than the target reliability for the tension resistance, since $n_{3}>n_{1}$.

\section{Uniformly Loaded Beam with Two Potential Failure Modes}

A simply supported uniformly loaded I-beam is studied in example problem 12.28 by Kachurin [7]. The steel $(E=210 G P a, G=80 G P a)$ beam has a length $l=8 \mathrm{~m}$ and the nominal cross section is characterised by (see Figure 5): $t_{w}=0.012 m, t_{f}=0.0178 m, b=0.19 m$ and $d=0.6 m$.

Two possible failure modes are considered: the first one being the maximum stress exceeding the allowable stress and the second one being the loading causing lateral buckling of the beam. To avoid these failures following conditions are set forth:

$$
\begin{gathered}
\sigma_{\max }=\frac{q l^{2} d}{16 I_{x}} \leq \sigma_{\text {all }}=\frac{\sigma_{y}}{n_{1}} \\
q \leq q_{\text {all }}=\frac{q_{\max }}{n_{3}}=\frac{1}{n_{3}} \gamma \frac{\sqrt{E I_{y} G J}}{l^{3}}
\end{gathered}
$$


with $q$ the uniform distributed load, $I_{x}$ and $I_{y}$, the area moment of inertia about the neutral axis parallel to $x$ and $y$, respectively, $J$ the torsional constant of the section and $\gamma$ a numerical constant. The formula for $q_{\max }$ and the look-up table for $\gamma$ is found in the standard book by Timoshenko [12].

\subsection{Random Dimension $t_{f}$}

In the following, the dimension $t_{f}$ is considered random. To simplify the calculations the cross-sectional dimensions are non-dimensionalised with respect to the nominal $t_{f}$ defined above, as a result the whole cross section will scale with the random $t_{f}$. The reliability of the structure can thus be calculated as follows:

$$
R=\operatorname{Prob}\left\{\left(\sigma_{\max } \leq \sigma_{y}\right) \cap\left(q \leq q_{\max }\right)\right\}
$$

in terms of the random $t_{f}$ and taking into account the non-dimensional quantities Eq. (74) becomes:

$$
R=\operatorname{Prob}\left\{\left(\sqrt[3]{\frac{q l^{2}}{3561.1 \sigma_{y}}} \leq t_{f}\right) \cap\left(\sqrt[4]{\frac{q}{\gamma} \frac{l^{3}}{45.9 \sqrt{E G}}} \leq t_{f}\right)\right\}
$$

The following three cases can be encountered:

1. $\sqrt[3]{\frac{q l^{2}}{3561.1 \sigma_{y}}}>\sqrt[4]{\frac{q}{\gamma} \frac{l^{3}}{45.9 \sqrt{E G}}}$, then the reliability is dominated by the yield constraint.

2. $\sqrt[4]{\frac{q}{\gamma} \frac{l^{3}}{45.9 \sqrt{E G}}}>\sqrt[3]{\frac{q l^{2}}{3561.1 \sigma_{y}}}$, then the reliability is dominated by the lateral buckling constraint.

3. $\sqrt[3]{\frac{q l^{2}}{3561.1 \sigma_{y}}}=\sqrt[4]{\frac{q}{\gamma} \frac{l^{3}}{45.9 \sqrt{E G}}}$, then either of the constraints can be used to find the reliability.

As above, a uniform distribution for the random variable, in this case $t_{f}$, is assumed. The reliability for the first case is calculated as

$$
R_{1}= \begin{cases}0 & \text { if } \sqrt[3]{\frac{q l^{2}}{3561.1 \sigma_{y}}}>h=E\left[t_{f}\right]+\sqrt{3} d_{t_{f}} \\ 1-\frac{\sqrt[3]{\frac{q l^{2}}{3561.1 \sigma_{y}}}-E\left[t_{f}\right]+\sqrt{3} d_{t_{f}}}{2 \sqrt{3} d_{t_{f}}} & \text { if } g \leq \sqrt[3]{\frac{q l^{2}}{3561.1 \sigma_{y}}} \leq h \\ 1 & \text { if } \sqrt[3]{\frac{q l^{2}}{3561.1 \sigma_{y}}}<g=E\left[t_{f}\right]-\sqrt{3} d_{t_{f}}\end{cases}
$$

The reliability for the second case is calculated as

$$
R_{2}= \begin{cases}0 & \text { if } \sqrt[4]{\frac{q}{\gamma} \frac{l^{3}}{45.9 \sqrt{E G}}}>h=E\left[t_{f}\right]+\sqrt{3} d_{t_{f}} \\ 1-\frac{\sqrt[4]{\frac{q}{\gamma} \frac{l^{3}}{45.9 \sqrt{E G}}}-E\left[t_{f}\right]+\sqrt{3} d_{t_{f}}}{2 \sqrt{3} d_{t_{f}}} & \text { if } g \leq \sqrt[4]{\frac{q}{\gamma} \frac{l^{3}}{45.9 \sqrt{E G}}} \leq h \\ 1 & \text { if } \sqrt[4]{\frac{q}{\gamma} \frac{l^{3}}{45.9 \sqrt{E G}}}<g=E\left[t_{f}\right]-\sqrt{3} d_{t_{f}}\end{cases}
$$


The mean of the maximum stress is found to be

$$
E\left[\Sigma_{\max }\right]=\frac{q l^{2}}{3561.1} E\left[\frac{1}{t_{f}^{3}}\right]=\frac{q l^{2}}{3561.1} \frac{1}{\left(E\left[t_{f}\right]\right)^{3}-6 E\left[t_{f}\right] d_{t_{f}}^{2}+9 \frac{d_{t_{f}}^{4}}{E\left[t_{f}\right]}}
$$

multiplying the numerator and denominator of the middle part of Eq. (76) by $\sqrt[3]{E\left[\Sigma_{\max }\right]}$ gives

$$
R_{1}=1-\frac{\sqrt[3]{\frac{q l^{2}}{3561.1 \sigma_{y}} E\left[\Sigma_{\max }\right]}-E\left[t_{f}\right] \sqrt[3]{\Sigma_{\max }}+\sqrt{3} d_{t_{f}} \sqrt[3]{\Sigma_{\max }}}{2 \sqrt{3} d_{t_{f}} \sqrt[3]{\Sigma_{\max }}}
$$

Taking into account the definition of the central safety factor (21), but with the random variable of interest now $\Sigma_{\max }$ instead of $\Sigma$, we get:

$$
\begin{aligned}
& R_{1}=1-\frac{\sqrt[3]{\frac{1}{n_{1}}}-\sqrt[3]{\frac{1}{Q}}+\sqrt[3]{\frac{3 \sqrt{3} \nu_{t_{f}}}{Q}}}{\sqrt[3]{\frac{24 \sqrt{3} \nu_{t_{f}}^{3}}{Q}}}, \\
& Q=\left[\frac{3561.1}{q l^{2}} E\left[\Sigma_{\max }\right]\left(E\left[t_{f}\right]\right)^{3}\right]^{-1}=1-6 \nu_{t_{f}}+9 \nu_{t_{f}}^{4}
\end{aligned}
$$

The expression for the safety factor $n_{1}$ as a function of the target reliability $r_{1}$ is

$$
n_{1}=\left[\left(1-r_{1}\right) \sqrt[3]{\frac{24 \sqrt{3} \nu_{t_{f}}^{3}}{Q}}+\sqrt[3]{\frac{1}{Q}}-\sqrt[3]{\frac{3 \sqrt{3} \nu_{t_{f}}^{3}}{Q}}\right]^{-3}
$$

To calculate the reliability $R_{2}$ as a function of $n_{3}$, first the mean of the maximum distributed load $E\left[q_{\max }\right]$ is calculated as

$$
\begin{aligned}
E\left[q_{\max }\right] & =45.9 \gamma \frac{\sqrt{E G}}{l^{3}} E\left[t_{f}^{4}\right] \\
& =45.9 \gamma \frac{\sqrt{E G}}{l^{3}} \frac{1}{10 \sqrt{3}}\left[10\left(E\left[t_{f}\right]\right)^{4} \sqrt{3}+60\left(E\left[t_{f}\right]\right)^{2} \sqrt{3} d_{t_{f}}^{2}+18 \sqrt{3} d_{t_{f}}^{4}\right]
\end{aligned}
$$

Secondly, we rewrite Eq. (77) in the form

$$
R_{2}=1-\frac{\sqrt[4]{\frac{q}{\gamma} \frac{l^{3}}{45.9 \sqrt{E G}} \frac{1}{E\left[q_{\max }\right]}}-E\left[t_{f}\right] \sqrt[4]{\frac{1}{E\left[q_{\max }\right]}}+\sqrt{3} d_{t_{f}} \sqrt[4]{\frac{1}{E\left[q_{\max }\right]}}}{2 \sqrt{3} d_{t_{f}} \sqrt[4]{\frac{1}{E\left[q_{\max }\right]}}}
$$

Taking into account following definition of the central safety factor

$$
n_{3}=\frac{E\left[q_{\max }\right]}{q}
$$


we can write

$$
\begin{aligned}
R_{2} & =1-\frac{\sqrt[4]{\frac{1}{n_{3}}}-\sqrt[4]{\frac{1}{P}}+\sqrt[4]{\frac{9 \nu_{t_{f}}^{4}}{P}}}{P}=\frac{\sqrt[4]{\frac{14 \nu_{t_{f}}^{4}}{P}}}{\left(E\left[t_{f}\right]\right)^{4}} \frac{l^{3}}{45.9 \gamma \sqrt{E G}}=\frac{1}{5}\left(5+30 \nu_{t_{f}}^{2}+9 \nu_{t_{f}}^{4}\right)
\end{aligned}
$$

The expression for the safety factor $n_{3}$ as a function of the target reliability $r_{2}$ is

$$
n_{3}=\left[\left(1-r_{2}\right) \sqrt[4]{\frac{144 \nu_{t_{f}}^{4}}{P}}+\sqrt[4]{\frac{1}{P}}-\sqrt[4]{\frac{9 \nu_{t_{f}}^{4}}{P}}\right]^{-4}
$$

It is important to note the similarities between the above equations (85), (86) and the equations (27), (28) and (53), (54) derived in the above sections. The similarity arises from the fact that the safety factors in all the three cases depends on the different random variables in the same way, namely with a power 4. This will be discussed further in section 6 .

If the same reliability is demanded for all constraints then the following relationship between $n_{1}$ and $n_{3}$ is found:

$$
n_{1}=\left[\left(\sqrt[4]{\frac{1}{n_{3}}}-\sqrt[4]{\frac{1}{P}}+\sqrt[4]{\frac{9 \nu_{t_{f}}^{4}}{P}}\right) \frac{\sqrt[3]{\frac{24 \sqrt{3} \nu_{t_{f}}^{3}}{Q}}}{\sqrt[4]{\frac{144 \nu_{t_{f}}^{4}}{P}}}+\sqrt[3]{\frac{1}{Q}}-\sqrt[3]{\frac{3 \sqrt{3} \nu_{t_{f}}^{3}}{Q}}\right]^{-3}
$$

or equivalently

$$
n_{3}=\left[\left(\sqrt[3]{\frac{1}{n_{1}}}-\sqrt[3]{\frac{1}{Q}}+\sqrt[3]{\frac{3 \sqrt{3} \nu_{t_{f}}}{Q}}\right) \frac{\sqrt[4]{\frac{144 \nu_{t_{f}}^{4}}{P}}}{\sqrt[3]{\frac{24 \sqrt{3} \nu_{t_{f}}^{3}}{Q}}}+\sqrt[4]{\frac{1}{P}}-\sqrt[4]{\frac{9 \nu_{t_{f}}^{4}}{P}}\right]^{-4}
$$

Let us consider a numerical example. Following numerical values are adopted: $\sigma_{y}=800 \mathrm{MPa}, \gamma=38$ and $q=18000 \mathrm{~N} / \mathrm{m}$. Assume the lower bound $g=$ $0.007 \mathrm{~m}$ and the upper bound $h=0.018 \mathrm{~m}$ for the uniform distribution of $t_{f}$. Then the following values are calculated:

$$
\begin{aligned}
E\left[t_{f}\right] & =0.0125 \mathrm{~m} \\
d_{t_{f}} & =0.00318 \mathrm{~m} \\
\nu_{t_{f}} & =0.254
\end{aligned}
$$

If one would demand the same reliability 0.9 for both failure modes, then the required safety factors would be $n_{1}=2.39$ and $n_{3}=7.91$. For a target reliability equal to 0.99 the required safety factors turn out to be $n_{1}=3.53$ and $n_{3}=13.32$. In the original example in [7] the safety factor $n_{3}$ suggested by Kachurin equals $n_{3}=1.7$. For the considered random $t_{f}$ this would mean a very low reliability of 0.55 , which is unacceptable. 


\section{Discussion}

In our analyses above, we sought to bridge the gap between safety factors living on their respective islands, as it were, of distinct failure modes. We showed that based on a common target reliability a clear relationship between these different safety factors can be established. The source of the randomness in the problem together with the definitions of the central safety factors determined the particular kind of the relationship. In the truss problem with the random $F$, all safety factors depended linearly on the random $F$, albeit with different scaling factors. These scaling factors however cancel out in the expressions of the safety factor as a function of the reliability. In both problems where the random $b$ was considered, the safety factor for the tension and displacement constraint depend both on the random $b$ in a quadratic way, whereas the safety factor for the buckling constraint depends on the dimension $b$ with a power 4 . This difference results in the different values of $n_{1}, n_{2}$ on the one hand and $n_{3}$ on the other hand as expressed in Eq. (55). The already noted similarity between the three sets of equations (85), (86) and (27), (28) and (53), (54) also stems from the fact the safety factor $n_{3}$ depends on the respective random variables in the same way, namely with a power 4 .

\section{Conclusion}

The assignment of safety factors related to different failure modes is studied in this paper using three simple (academic) examples. It was shown that a consistent assignment is possible through the use of the probabilistic reliability concept. In particular, the origin of the randomness in the design and its relation to each central safety factor determines the relation between the safety factors. This relation varies in the studied examples from equality of safety factors to polynomial relations with fractional powers. Although this work is concentrated on simple (academic) examples, it can be interesting for engineering practice and teaching. However, to highlight the scientific relevance of the presented approach and to demonstrate the field of application, more complicated structures have to be investigated. Appropriate analysis is underway and will be published elsewhere.

\section{Acknowledgment}

This study was performed during the research stay of W.V. at the Florida Atlantic University as a Fulbright scholar. W.V. would like to acknowledge the highly appreciated support of the IWT-Flanders, the Commission for Educational Exchange Between The United States of America, Belgium and Luxembourg (The Fulbright Fund and Vesuvius) and the Raymond Snoeys fund of the KU Leuven.

The authors would like to thank the reviewers for their valuable comments. 


\section{Appendix}

Random dimension b, quadratic type of probability density function

The procedure for linking the two safety factors in section 3.1 can be applied for probability distributions different from the uniform one. In this section the guidelines for dealing with a quadratic type probability density function with bounds $g$ and $h$ are given. Please note that towards the end of the procedure not all expressions are explicitely reported. Let $b$ have a probability density function of the form:

$$
f_{b}= \begin{cases}0 & \text { if } b<g \text { or } b>h \\ \frac{6}{(g-h)^{3}} b^{2}+\frac{-6(g+h)}{(g-h)^{3}} b+\frac{6 g h}{(g-h)^{3}} & \text { if } g \leq b \leq h\end{cases}
$$

so that the mean equals $E[b]=\int_{g}^{h} b f_{b}(b) \mathrm{d} b=(g+h) / 2$, and the standard deviation is

$$
d_{b}=\sqrt{E\left[(b-E[b])^{2}\right]}=\sqrt{\int_{g}^{h}\left(b-\frac{g+h}{2}\right)^{2} f_{b}(b) \mathrm{d} b}=\frac{h-g}{2 \sqrt{5}}
$$

The lower and upper bound on $b$ can be described by the mean and the standard deviation: $g=E[b]-\sqrt{5} d_{b}$ and $\mathrm{h}=E[b]+\sqrt{5} d_{b}$. Furthermore, the probability density function Eq. (92) for values between $g$ and $h$ can be rewritten as function of $E[b]$ and $d_{b}$ :

$$
f_{b}(b)=\frac{-3 \sqrt{5} b^{2}}{100 d_{b}^{3}}+\frac{3 E[b] \sqrt{5} b}{50 d_{b}^{3}}-\frac{3 \sqrt{5}\left((E[b])^{2}-5 d_{b}^{2}\right)}{100 d_{b}^{3}}
$$

The reliability for the first case is thus calculated as

$$
R_{1}=\left\{\begin{array}{cl}
0 & \text { if } \sqrt{\frac{P}{3 \sigma_{y}}}>h=E[b]+\sqrt{5} d_{b} \\
1-\frac{\sqrt{5}}{100 d_{b}^{3}}\left\{-\left(\sqrt{\frac{P}{3 \sigma_{y}}}\right)^{3}+\frac{E[b] P}{\sigma_{y}}-3(E[b])^{2} \sqrt{\frac{P}{3 \sigma_{y}}} \ldots\right. & \\
\left.+15 d_{b}^{2} \sqrt{\frac{P}{3 \sigma_{y}}}+(E[b])^{3}+10 \sqrt{5} d_{b}^{3}-15 E[b] d_{b}^{2}\right\} & \text { if } g \leq \sqrt{\frac{P}{3 \sigma_{y}}} \leq h \\
1 & \text { if } \sqrt{\frac{P}{3 \sigma_{y}}}<g=E[b]-\sqrt{5} d_{b}
\end{array}\right.
$$


The reliability for the second case is evaluated as

$$
R_{2}= \begin{cases}0 & \text { if } \sqrt[4]{\frac{P 4 l^{2}}{\pi^{2} E}}>h=E[b]+\sqrt{5} d_{b} \\ 1-\frac{\sqrt{5}}{100 d_{b}^{3}}\left\{-\left(\sqrt[4]{\frac{P 4 l^{2}}{\pi^{2} E}}\right)^{3}+3 E[b] \sqrt[2]{\frac{P 4 l^{2}}{\pi^{2} E}}-3(E[b])^{2} \sqrt[4]{\frac{P 4 l^{2}}{\pi^{2} E}} \ldots\right. & \\ \left.+15 d_{b}^{2} \sqrt[4]{\frac{P 4 l^{2}}{\pi^{2} E}}+(E[b])^{3}+10 \sqrt{5} d_{b}^{3}-15 E[b] d_{b}^{2}\right\} & \text { if } g \leq \sqrt[4]{\frac{P 4 l^{2}}{\pi^{2} E}} \leq h \\ 1 & \text { if } \sqrt[4]{\frac{P 4 l^{2}}{\pi^{2} E}}<g=E[b]-\sqrt{5} d_{b}\end{cases}
$$

The mean value of the stress is

$$
E[\Sigma]=\frac{P}{3} E\left[\frac{1}{b^{2}}\right]=\frac{P}{3} \frac{-3}{50 d_{b}^{3}}\left\{E[b] \sqrt{5}\left[\ln \left(E[b]-\sqrt{5} d_{b}\right)-\ln \left(E[b]+\sqrt{5} d_{b}\right)\right]+10 d_{b}\right\}
$$

since

$$
\begin{aligned}
E\left[\frac{1}{b^{2}}\right] & =\int_{E[b]-\sqrt{5} d_{b}}^{E[b]+\sqrt{5} d_{b}} \frac{1}{b^{2}}\left(\frac{-3 \sqrt{5} b^{2}}{100 d_{b}^{3}}+\frac{3 E[b] \sqrt{5} b}{50 d_{b}^{3}}-\frac{3 \sqrt{5}\left[(E[b])^{2}-5 d_{b}^{2}\right]}{100 d_{b}^{3}}\right) \mathrm{d} b \\
& =\frac{-3}{50 d_{b}^{3}}\left\{E[b] \sqrt{5}\left[\ln \left(E[b]-\sqrt{5} d_{b}\right)-\ln \left(E[b]+\sqrt{5} d_{b}\right)\right]+10 d_{b}\right\}
\end{aligned}
$$

with the expression between brackets on the first line of the above equation being equal to the probability density function as reported in Eq. (94).

Multiplying the numerator and denominator of the middle part of Eq. (95) by $(\sqrt{E[\Sigma]})^{3}$ yields

$$
\begin{aligned}
R_{1} & =1-\frac{\sqrt{5}}{100 d_{b}^{3}(\sqrt{E[\Sigma]})^{3}}\left\{-\left(\sqrt{\frac{P}{3 \sigma_{y}}}\right)^{3}(\sqrt{E[\Sigma]})^{3}+\frac{E[b] P}{\sigma_{y}}(\sqrt{E[\Sigma]})^{3} \ldots\right. \\
& -3(E[b])^{2} \sqrt{\frac{P}{3 \sigma_{y}}}(\sqrt{E[\Sigma]})^{3}+15 d_{b}^{2} \sqrt{\frac{P}{3 \sigma_{y}}}(\sqrt{E[\Sigma]})^{3}+(E[b])^{3}(\sqrt{E[\Sigma]})^{3} \ldots \\
& \left.+10 \sqrt{5} d_{b}^{3}(\sqrt{E[\Sigma]})^{3}-15 E[b] d_{b}^{2}(\sqrt{E[\Sigma]})^{3}\right\}
\end{aligned}
$$

Taking into account Eq. (21) and noting again that the coefficient of variation 
of $b$ is $\nu_{b}=d_{b} / E[b]$, the above equation becomes

$$
\begin{aligned}
R_{1} & =1-\frac{10 \sqrt{5} \nu^{3 / 2}}{Q^{3 / 2}}\left[-\left(\sqrt{\frac{P}{3 n_{1}}}\right)^{3}+\frac{P Q^{1 / 2}}{10 n_{1} \nu^{3 / 2}} \ldots\right. \\
& -\frac{\sqrt{3}}{100 \nu^{3}} \sqrt{\frac{P}{n_{1}}} Q+\frac{\sqrt{3}}{20 \nu} \sqrt{\frac{P}{n_{1}}} Q+\frac{1}{1000 \nu^{9 / 2}} Q^{3 / 2} \ldots \\
& \left.+\frac{\sqrt{5}}{100 \nu^{3 / 2}} Q^{3 / 2}-\frac{3}{200 \nu^{5 / 2}} Q^{3 / 2}\right]
\end{aligned}
$$

with

$$
Q=-2 P\left[\sqrt{5} \ln \left(\frac{1-\sqrt{5} \nu}{1+\sqrt{5} \nu}\right)+10 \nu\right]
$$

From this equation one can solve for $n_{1}$ as a function of the target reliability $r_{1}$ leading to $n_{1}\left(r_{1}\right)$ which is not written down here. The remainder of the calculation requires to compute

$$
\begin{aligned}
E\left[N_{\text {critical }}\right] & =E\left[\frac{\pi^{2} E b^{4}}{4 l^{2}}\right]=\frac{\pi^{2} E}{4 l^{2}} E\left[b^{4}\right] \\
& =\frac{\pi^{2} E}{4 l^{2}}\left[(E[b])^{4}+6(E[b])^{2} d_{b}^{2}+\frac{15}{7} d_{b}^{4}\right]
\end{aligned}
$$

When dividing the numerator and denominator of the middle part in Eq. (96) by $\left(\sqrt[4]{E\left[N_{\text {critical }}\right]}\right)^{3}$ one finds an expression of $R_{2}$ as a function of $n_{3}$, with $n_{3}$ defined as in Eq. (26). For space limitation reasons the expressions are no longer reported. The expression $R_{2}\left(n_{3}\right)$ can in turn be transformed to yield $n_{3}$ as a function of a target reliability $r_{2}: n_{3}\left(r_{2}\right)$. By postulating that the structure should possess the uniform reliability against each mode of failure, namely $r_{1}=r_{2}$, then from $r_{1}\left(n_{1}\right)=r_{2}\left(n_{3}\right)$ one can derive the expression relating $n_{1}$ and $n_{3}$. 


\section{References}

[1] Ambrose, J. and Tripeny, P. Simplified Design of Steel Structures. Wiley \& Sons, New Jersey, 2007.

[2] Chen, W.F. and Lui, E.M. Stability Design Criteria for Steel Members and Frames in the United States. Journal of Constructional Steel Research, 5:31-74, 1985 .

[3] Elishakoff, I. Safety Factors and Reliability: Friends or Foes?, Kluwer Academic Publishers, Dordrecht, 2004.

[4] Ellingwood, B.R. LRFD: implementing structural reliability in professional practice, Engineering Structures, 22:106-115, 2000.

[5] Haldar, A. and Mahadevan, S. Probability, reliability, and statistical methods in engineering design. Wiley, 2000.

[6] Izkovich, G.M. Strength of Materials. "Visshaya Shkola" Publishers, Moscow, 2001 (in Russian).

[7] Kachurin, V.P., editor. Collection of Strength of Materials Problems. "Nauka" Publishers, Moscow, 1970 (in Russian).

[8] Maquoi, R. and Rondal, J. Analytical Formulation of the New European Buckling Curves. Brussels, centre Belgo-Luxembourgeois d'information de l'acier, 1978.

[9] Miroliubov, I.N. and Engalychev, C.A. and Sergievski, N.D. and Almametov, F.Z. and Kuritsyn, N.A. and Smirnov-Vasiliev, K.G. and Yashina, L.V. Guide to solve the Strength of Materials Problems. "Visshaya Shkola" Publishers, Moscow, 1967 (in Russian).

[10] Thoft-Christensen, P. and Baker, M.J. Structural reliability theory and its applications. Springer, 1982.

[11] Thoft-Christensen, P. and Murotsu, Y. Application of structural systems reliability theory. Springer, 1986.

[12] Timoshenko, S.P. and Gere, J.M. Theory of Elastic Stability. McGraw-Hill, 1982.

[13] Vinokurov, A. Collection of Problems in Strength of Materials. Moscow, 1990 (in Russian).

[14] Volmir, A.S., editor. Collection of Problems in Strength of Materials. "Nauka" Publishers, Moscow, 1984 (in Russian). 